Article

\title{
Investigating the Early Events after Skin-Barrier Disruption Using Microdialysis-A Human Ex Vivo Skin Model
}

\author{
Katrine Baumann ${ }^{1,2, * \mathbb{D}}$, Niels Peter Hell Knudsen ${ }^{1}$, Anne-Sofie Østergaard Gadsbøll ${ }^{2} \mathbb{D}$, Anders Woetmann ${ }^{2} \mathbb{C}$ \\ and Per Stahl Skov 1,3 \\ 1 RefLab ApS, DK-2200 Copenhagen, Denmark; nielsphk@gmail.com (N.P.H.K.); pss@reflab.dk (P.S.S.) \\ 2 LEO Foundation Skin Immunology Research Center, Department of Immunology and Microbiology, \\ University of Copenhagen, DK-2200 Copenhagen, Denmark; asgadsboll@sund.ku.dk (A.-S.Ø.G.); \\ awoetmann@sund.ku.dk (A.W.) \\ 3 Odense Research Center for Anaphylaxis (ORCA), Department of Dermatology and Allergy Center, \\ Odense University Hospital, DK-5000 Odense, Denmark \\ * Correspondence: kybaumann@gmail.com; Tel.: +45-7070-2345
}

Citation: Baumann, K.; Knudsen, N.P.H.; Gadsbøll, A.-S.Ø.; Woetmann, A.; Skov, P.S. Investigating the Early Events after Skin-Barrier Disruption Using Microdialysis-A Human Ex Vivo Skin Model. Dermato 2021, 1, 47-58. https://doi.org/10.3390/ dermato1020008

Academic Editor: Francois Niyonsaba

Received: 14 September 2021

Accepted: 19 November 2021

Published: 30 November 2021

Publisher's Note: MDPI stays neutral with regard to jurisdictional claims in published maps and institutional affiliations.

Copyright: (c) 2021 by the authors. Licensee MDPI, Basel, Switzerland. This article is an open access article distributed under the terms and conditions of the Creative Commons Attribution (CC BY) license (https:// creativecommons.org/licenses/by/ $4.0 /)$.

\begin{abstract}
Skin-barrier restoration following abrasive trauma is facilitated by mediator release from skin-resident cells, a process that has been investigated primarily in mice or simplified human systems with previous studies focusing on a limited number of biomarkers. Here, we demonstrate how early events caused by skin-barrier disruption can be studied in a human ex vivo skin model. Ten relevant biomarkers were recovered from the interstitial fluid by skin microdialysis with subsequent sample analysis using a multiplex platform. As a control, the biomarker profiles obtained from microdialysis sampling were compared to profiles of skin biopsy homogenates. We found that nine (GM-CSF, CXCL1/GRO $\alpha$, CXCL8/IL-8 CXCL10/IP-10, IL-1 $\alpha$, IL-6, MIF, TNF- $\alpha$, and VEGF) of the 10 biomarkers were significantly upregulated in response to abrasive trauma. Only dialysate levels of CCL27/CTACK were unaffected by skin abrasion. Biomarker levels in the homogenates corresponded to dialysate levels for CCL27/CTACK, CXCL1/GRO $\alpha$, CXCL8/IL-8, and IL-6. However, IL- $1 \alpha$ showed an inverse trend in response to trauma, and biopsy levels of MIF were unchanged. GM-CSF, CXCL10/IP-10, TNF- $\alpha$, and VEGF were not detected in the biopsy homogenates. Our results suggest that the human ex vivo skin model is a reliable approach to study early events after disruption of the skin barrier.
\end{abstract}

Keywords: biomarkers; cytokines; microdialysis; skin barrier; skin models; wound healing; ex vivo

\section{Introduction}

The skin is the largest human organ, and it serves as an important outer barrier against harmful elements in our environment, e.g., sunlight, pathogens, and mechanical trauma, in addition to being an important immunological organ which also has neuroendocrine functions [1]. A breach of the skin barrier compromises the body's first line of defense, making a quick and effective repair of the barrier vital. Several cell types are implicated in barrier restoration through secretion of signaling molecules such as cytokines and growth factors [2]. A timely and controlled release of these mediators is important to avoid disturbances in the barrier physiology, which makes these molecules highly relevant readouts when investigating barrier-disrupted skin $[2,3]$.

Several noninvasive methods have been used to study barrier function, e.g., measurements of trans-epidermal water loss, laser scanning microscopy, and Raman spectroscopy [4,5], but these approaches are unable to assess the cellular responses to barrier disruption in terms of mediator release. In contrast, skin microdialysis enables the extraction of soluble biomarkers released into the interstitial fluid in a minimally invasive manner. Thus, microdialysis sampling facilitates real-time monitoring of cutaneous events through recovery of endogenous and exogenous molecules from the target organ $[6,7]$. 
Punch biopsies may serve as an alternative approach by facilitating the identification of biomarkers via immunohistochemistry or tissue homogenization, followed by gene expression analysis [8]. However, as opposed to skin microdialysis that allows for continuous sampling, biopsies only provide brief snapshots of continuous processes and are considered more invasive in nature [9].

Studies of skin-barrier homeostasis are often carried out using animal models, which are easily accessible, but they do not accurately portray all aspects of the human organ [10-12]. Clinical studies, on the other hand, are expensive and time-consuming to conduct, which highlights the need for suitable human models to investigate the cellular responses to skin-barrier disruption.

Excised human skin has previously been used to examine regeneration of the outer skin layer, stratum corneum, after cyanoacrylate skin surface stripping [13-15] and the release of biomarkers from skin-resident cells after perturbation of the barrier [16,17]. Yet, these studies focused on a very limited number of biomarkers (e.g., interleukin (IL)-6 and IL-8 assessed by Döge et al. [16]) or an isolated cutaneous compartment (e.g., epidermal biomarker expression studied by Pfannes et al. [17]).

In this paper, we present an alternative model to study the early responses (i.e., within the first $24 \mathrm{~h}$ ) to skin-barrier disruption by subjecting human ex vivo skin to abrasive trauma, followed by an extensive biomarker profiling of the interstitial fluid using microdialysis sampling (Figure 1). Furthermore, analysis of human skin biopsy homogenates was included to compare biomarker profiles between the two methods.

a

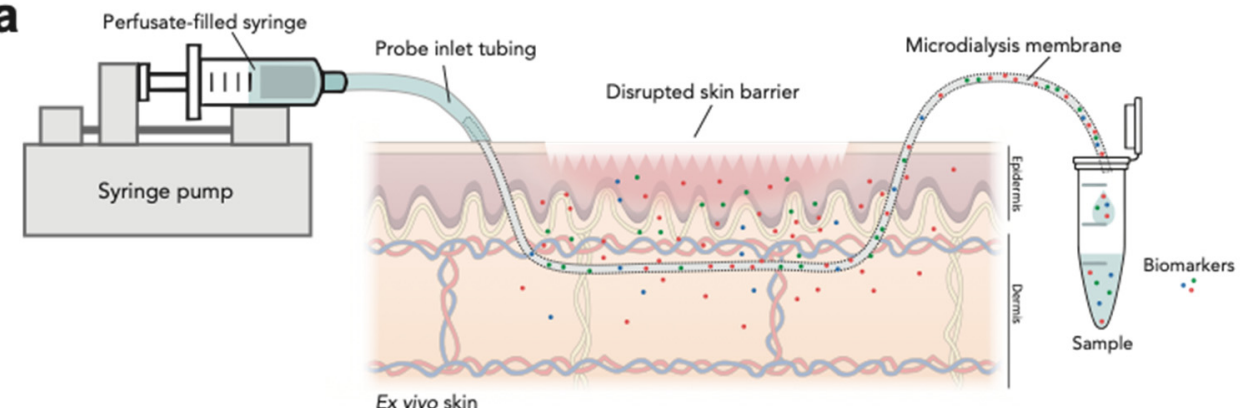

b

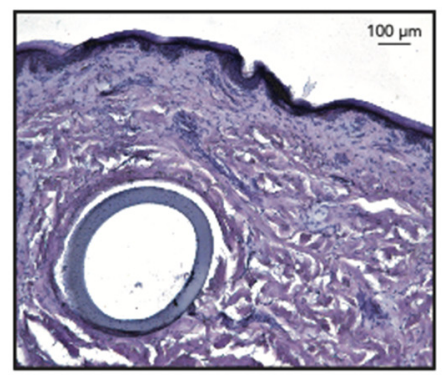

Figure 1. The human ex vivo skin model. (a) A schematic representation of the experimental model used to study acute changes in biomarker levels after disruption of the epidermal barrier on human ex vivo skin specimens. Abrasive trauma is applied to excised human skin, followed by sampling of biomarkers released from the skin-resident cells using microdialysis. Individual elements are not depicted to scale. (b) Cross-section of a microdialysis membrane within the skin. Scale bar: $100 \mu \mathrm{m}$.

\section{Materials and Methods}

\subsection{Skin Specimens}

Human abdominal skin was obtained from individuals undergoing cosmetic surgery $(n=10)$. Due to anonymization of the donors, the skin was obtained and used in this study in accordance with the Danish Act on Research Ethics Review of Health Research Projects, Section 14 (3) concerning the use of anonymized human material for research purposes and the Declaration of Helsinki.

\subsection{Skin Barrier Disruption}

The skin was prepared by mechanical removal of subcutaneous fat followed by excision of smaller skin specimens measuring approximately $4 \times 5 \mathrm{~cm}$, which were washed briefly in ethanol, antifungals, and sterile saline. Four skin specimens were prepared from each of the first five skin donors (D1-D5; used for proof-of-concept experiments), whereas two skin specimens were prepared from each of the following five donors (D6-D10; main experiments). Half of the skin specimens from each donor were subjected to abrasive trauma, while the other half were used as negative control (no abrasive trauma). Skin-barrier disruption was carried out by abrading the epidermal side 15 times back 
and forth with sandpaper (grit size 150), in line with previous studies [18-20]. In the proof-of-concept experiments (assessing IL-1 $\alpha$, IL-6, and MIF), two 21G cannulas were inserted into each skin specimen, whereas three guide cannulas were inserted in the main experiments (complete biomarker profiling). After abrasion, the skin specimens were incubated inside petri dishes on stainless-steel grids with culture medium underneath (DMEM supplemented with 5\% FCS, 2 mM GlutaMAX, 1\% penicillin/streptomycin, and $5 \mu \mathrm{g} / \mathrm{mL}$ recombinant human insulin) to simulate an air-liquid interface. The skin was incubated at $37{ }^{\circ} \mathrm{C}$ in humidified air with $5 \% \mathrm{CO}_{2}$ for 6 and $24 \mathrm{~h}$ (the latter only for in the proof-of-concept experiments) before microdialysis sampling was carried out. These timepoints were included to study early (but not acute) events associated with release of trauma-associated biomarkers.

\subsection{Microdialysis Setup}

Following incubation, the skin specimens were pinned dermal side down onto Styrofoam with a moist tissue paper in between to keep the tissue hydrated. Microdialysis probes with a $3000 \mathrm{kDa}$ molecular weight cutoff (Ultra High Flux, EP Medical, Copenhagen, Denmark) were inserted intradermally through the tip of the guide cannulas, which were then removed. To ensure proper flow, the entire setup was tilted at a $45^{\circ}$ angle before probe perfusion with Ringer lactate supplemented with $1 \%$ human albumin $+4 \mathrm{mM}$ lactic acid at a flow rate of $0.8 \mu \mathrm{L} / \mathrm{min}$. Sampling was carried out at room temperature for $2 \mathrm{~h}$ with continuous dialysate collection in PCR tubes covered with Parafilm to minimize evaporation. Fluid recovery was monitored gravimetrically, and samples were stored at $-80{ }^{\circ} \mathrm{C}$ until analysis.

\subsection{Protein Extraction from Punch Biopsies}

Three $3 \mathrm{~mm}$ punch biopsies were extracted from each skin specimen prior to microdialysis sampling at least $1.5 \mathrm{~cm}$ away from the inserted probes. Biopsies were stored at $-80{ }^{\circ} \mathrm{C}$ until homogenization of the tissue, which was performed using a Precellys Evolution homogenizer coupled to a Cryolys cooling system (Bertin Technologies, Montigny-le-Bretonneux, France) keeping the temperature at $\max 4{ }^{\circ} \mathrm{C}$. Triplicate biopsies (from the same skin specimen) were pooled in homogenizer vials (MK28-R, Bertin Technologies, Montigny-le-Bretonneux, France) and $1 \mathrm{~mL}$ of extraction solution (lysis buffer; Tris/Saline/EDTA buffer with 1\% Triton X-100 + cOmplete Protease Inhibitor cocktail, Roche, Basel, Switzerland) was added before the tissue was processed at $6800 \mathrm{rpm}$ for $8 \times 30 \mathrm{~s}$. Vials were spun at $20,000 \times g$ for $10 \mathrm{~min}$ at $4{ }^{\circ} \mathrm{C}$ before supernatants were extracted. The total protein concentration was determined for all extracts using the Bradford assay, and biopsy extracts were adjusted to a total protein concentration of $100 \mu \mathrm{g} / \mu \mathrm{L}$ prior to biomarker profiling using the enzymelinked immunosorbent assay (ELISA) kits listed below.

\subsection{Sample Analysis}

Dialysates from proof-of-concept experiments (skin donor D1-D5; assayed for IL- $1 \alpha$, IL-6, and MIF) and tissue homogenates (punch biopsies from skin donor D6-D10; assayed for all 10 biomarkers) were analyzed using commercially available ELISA kits from R\&D (Minneapolis, MN): Human CCL27/CTACK DuoSet (cat. no. DY376), human GM-CSF DuoSet (cat. no. DY215), human CXCL1/GRO $\alpha$ DuoSet (cat. no. DY275), human CXCL8/IL-8 DuoSet (cat. no. DY208), human CXCL10/IP-10 DuoSet (cat. no. DY266), human IL-1 $\alpha$ DuoSet (cat. no. DY200), human IL-6 DuoSet (cat. no. DY206), human MIF DuoSet (cat. no. DY289), human TNF- $\alpha$ DuoSet (cat. no. DY210), and human VEGF DuoSet (cat. no. DY293B). Duplicate dialysates from the proof-of-concept experiments (D1-D5) were pooled prior to quantification of IL- $1 \alpha$, IL- 6 , and MIF. Dialysates from the main experiments (D6-D10) were analyzed as individual samples (no pooling of samples from triplicate probes) in technical duplicates using the Meso Scale Discovery (MSD) U-PLEX assay. The 9-plex MSD assay was used for quantification of all biomarkers apart from MIF, which was analyzed separately by ELISA due to high tissue concentrations. 


\subsection{Histology of Microdialysis Membranes within the Skin}

A $6 \mathrm{~mm}$ skin biopsy was made perpendicular over an inserted microdialysis membrane and stored in formalin at room temperature overnight. Excess formalin was washed out by agitating the biopsy for $2 \mathrm{~h}$ in $0.9 \% \mathrm{NaCl}$ solution. The tissue was protected from freeze damage by incubating in a 30\% sucrose with $0.9 \% \mathrm{NaCl}$ solution for $10 \mathrm{~min}$ before it was embedded in optimal cutting temperature compound (OCT) compound (Agar Scientific Ltd., Stansted, UK) and sectioned into $30 \mu \mathrm{m}$ sections using a Cryotome ${ }^{\mathrm{TM}}$ FSE Cryostat (Thermo Scientific, Loughborough, UK). The cryosections were mounted on microscope slides and stained with hematoxylin and eosin to visualize placement of the microdialysis membrane inside the skin.

\subsection{Statistical Analysis}

Statistical analyses were conducted using GraphPad Prism version 8.2.1 (GraphPad Software, San Diego, CA, USA). Mean values of control and trauma groups were compared using the Wilcoxon matched-pairs signed rank test (one-tailed; the a priori hypothesis entailed increasing levels of trauma-associated biomarkers) due to the small sample size. Outliers were identified using the Grubbs' test. Biomarker levels above zero but below the MSD assay limit of quantification (defined as the lower limit of detection stated by the manufacturer) were set to the value of the lower limit of quantification (LLOQ). This value was also used for subsequent statistical analyses.

\section{Results}

A proof-of-concept study was carried out by assessing extracellular levels of three biomarkers associated with early phase tissue trauma in excised skin from five individual donors. IL-1 $\alpha$, IL-6, and macrophage migration inhibitory factor (MIF) were sampled by microdialysis 6 and $24 \mathrm{~h}$ after abrasive disruption of the skin barrier, to allow for de novo synthesis and release of biomarkers, and subsequently quantified in the dialysates using ELISA. All three biomarkers were significantly upregulated in the skin $6 \mathrm{~h}$ after the abrasive procedure compared with background levels detected in control skin specimens (Figure 2). After 24 h, levels of IL-1 $\alpha$, IL-6, and MIF were significantly above the levels measured in control specimens (Figure 2), despite concentrations of IL-1 $\alpha$ decreasing to near-background levels (Figure 2a). Levels of IL-6 were significantly higher in control specimens after $24 \mathrm{~h}(p=0.0312)$ (Figure $2 \mathrm{~b}$ ), whereas background levels of IL-1 $\alpha$ (Figure $2 \mathrm{a}$ ) and MIF (Figure 2c) did not change significantly from 6 to $24 \mathrm{~h}$ of incubation. Due to a decreasing signal-to-noise ratio from 6 to $24 \mathrm{~h}$ post barrier disruption, only the $6 \mathrm{~h}$ timepoint was included in the subsequent experiments. To assess the impact of skin incubation, tissue viability was assessed in response to incubation time by histological examination of skin specimens, showing that tissue integrity was well maintained after $24 \mathrm{~h}$ of incubation (Figure S1), and proliferating basal keratinocytes (assessed by Ki67 staining) persisted within the first $48 \mathrm{~h}$ of incubation (Figure S2).

Skin specimens were obtained from five new donors, and seven additional biomarkers were added to the panel, now comprising 10 different cytokines, chemokines, and growth factors associated with skin-barrier repair: chemokine ( $\mathrm{C}-\mathrm{C}$ motif) ligand 27 (CCL27)/cutaneous T-cell-attracting cytokine (CTACK), granulocyte-macrophage colonystimulating factor (GM-CSF), chemokine (C-X-C motif) ligand (CXCL)1/growth-regulated oncogene $\alpha(\mathrm{GRO} \alpha), \mathrm{CXCL} 8 / \mathrm{IL}-8, \mathrm{CXCL} 10 /$ interferon- $\gamma$-induced protein 10 (IP-10), IL-1 $\alpha$, IL-6, MIF, tumor necrosis factor- $\alpha$ (TNF- $\alpha)$, and vascular endothelial growth factor (VEGF). Biomarkers were collected from the skin specimens $6 \mathrm{~h}$ after abrasive disruption of the barrier using microdialysis. The dialysates were analyzed using the MSD multiplex platform instead of ELISA, as the MSD assay facilitated highly sensitive detection of multiple analytes in a single sample. Furthermore, the MSD platform is capable of analyzing smaller sample volumes, thereby enabling the extension of the biomarker panel along with measurements of individual samples (as opposed to pooling of samples prior to analysis). 
a
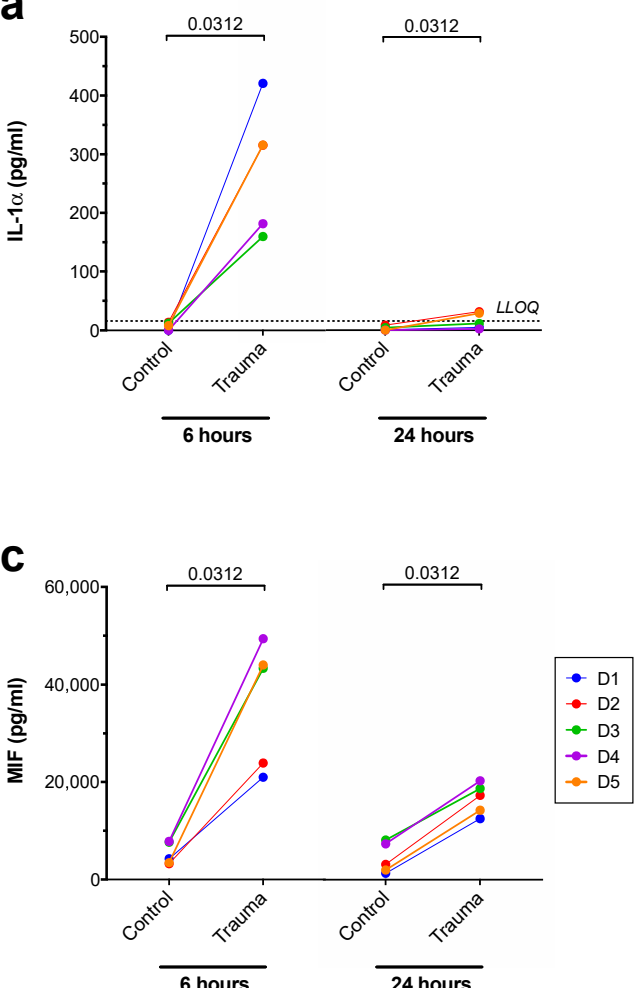

b

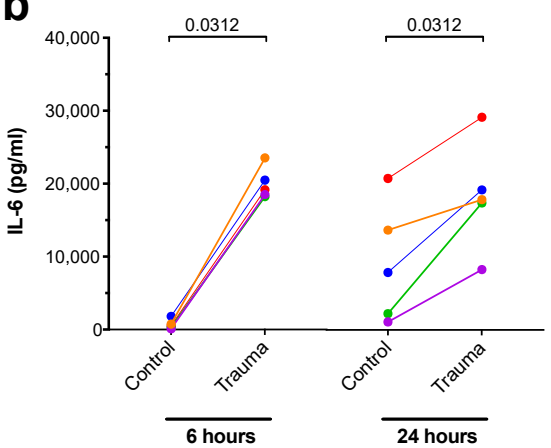

Figure 2. Dialysate levels of (a) IL-1 $\alpha$, (b) IL-6, and (c) MIF obtained from five individual skin donors and quantified by ELISA. Unaffected control and barrier-disrupted (trauma) skin specimens were prepared for each donor and incubated for 6 or $24 \mathrm{~h}$ prior to microdialysis sampling. Two probes were inserted into each skin specimen, and dialysates from duplicate probes were pooled prior to biomarker quantification. Numbers above the bars indicate $p$-values obtained from Wilcoxon matched-pairs signed rank test. LLOQ = lower limit of quantification (only depicted in graphs with measurements below LLOQ).

Nine of the 10 biomarkers were significantly upregulated in dialysates after skin barrier disruption across the five donors; GM-CSF, CXCL1/GRO $\alpha$, CXCL8/IL-8, CXCL10/IP10, IL-1 $\alpha$, IL-6, MIF, TNF- $\alpha$, and VEGF (Figure 3). Only levels of CCL27/CTACK were not significantly affected $6 \mathrm{~h}$ after disruption of the skin barrier (Figure 3a). Overall, the average intersubject variability in dialysate levels (measured as the coefficient of variation) was $61 \%$, whereas the average variability across replicate probes from the same skin specimen (the "intrasubject variability") was $23 \%$. The intrasubject variability might be further reduced in future studies if the insertion depth is guided by ultrasound scanning, but this was not performed as part of the current study due to lack of access to suitable equipment. However, it is generally accepted that consistent use of the same skilled operator leads to minimal variations in probe placement [1-3] (refer to Figure $1 \mathrm{~b}$ for an example of the intradermal positioning of a microdialysis membrane).

In order to compare microdialysis sampling with an alternative technique, three $3 \mathrm{~mm}$ punch biopsies were extracted from all skin specimens, immediately before microdialysis sampling was commenced, and then homogenized to determine total tissue concentrations (intracellular + extracellular) of the biomarkers (Figure 4). Six out of 10 biomarkers were detected in the homogenates; CCL27/CTACK, CXCL1/GRO $\alpha$, CXCL8/IL-8, IL-1 $\alpha$, IL-6, and MIF, whereas GM-CSF, CXCL10/IP-10, TNF- $\alpha$, and VEGF were not found in levels above the LLOQ (Figure 4 and Table 1). Homogenate concentrations of CXCL1/GRO $\alpha$ (Figure 4b), CXCL8/IL-8 (Figure 4c), and IL-6 (Figure 4e) were significantly increased in response to disruption of the skin barrier, whereas an outlier (D6) rendered the trend toward decreasing biopsy levels of IL- $1 \alpha$ insignificant (Figure $4 \mathrm{~d}$ ). The total tissue concentrations 
of CCL27/CTACK (Figure 4a) and MIF (Figure 4f) measured in the homogenates were not significantly affected by the abrasive procedure.

a

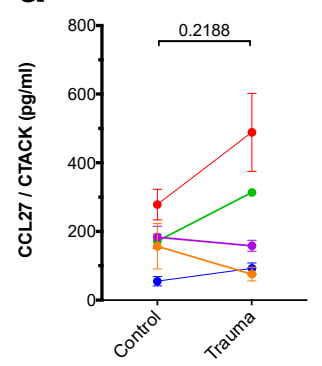

f

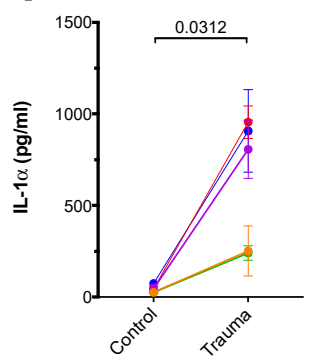

b

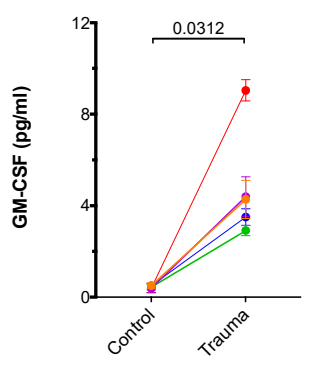

g

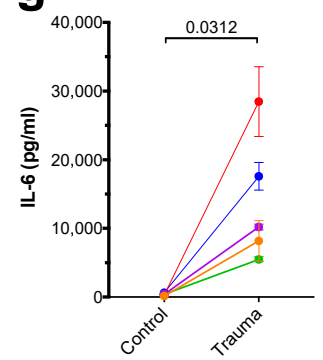

C

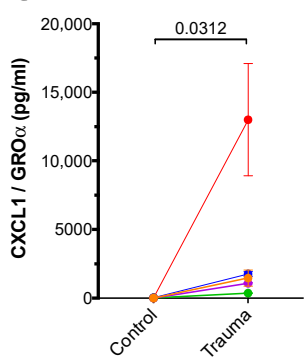

h

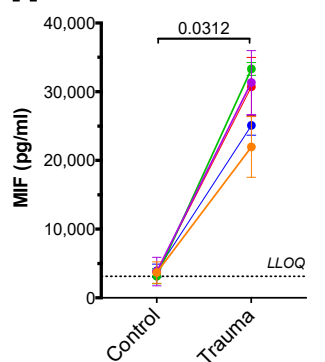

d

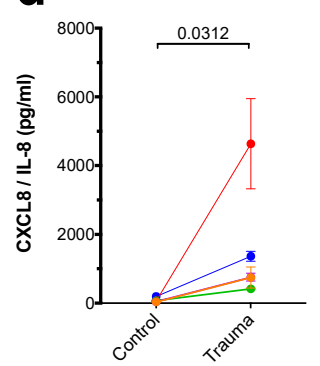

i

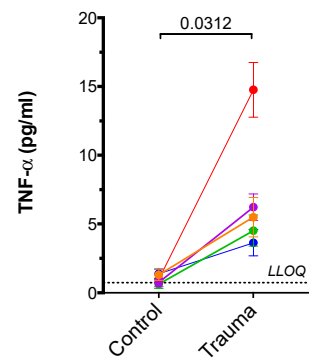

e

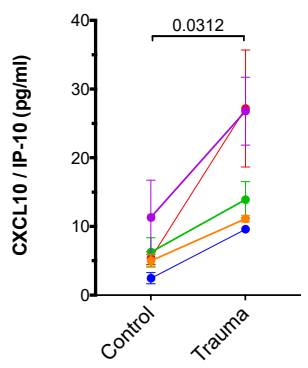

j

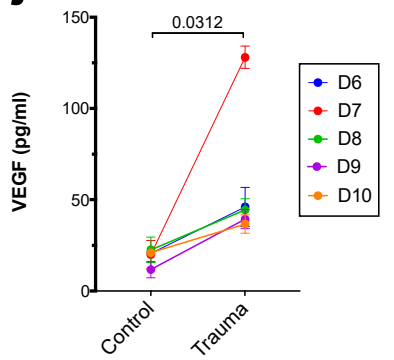

Figure 3. Dialysate levels of (a) CCL27/CTACK, (b) GM-CSF, (c) CXCL1/GRO $\alpha$, (d) CXCL8/IL-8, (e) CXCL10/IP-10, (f) IL-1 $\alpha$, (g) IL-6, (h) MIF, (i) TNF- $\alpha$, and (j) VEGF measured $6 \mathrm{~h}$ post abrasion using the MSD multiplex platform. Data are shown for barrier-disrupted skin specimens (trauma) or control specimens prepared from five individual skin donors and incubated for $6 \mathrm{~h}$. Depicted is the mean $\pm \mathrm{SD}$ of triplicate probes from each skin specimen. Numbers above the bars indicate $p$-values obtained from Wilcoxon matched-pairs signed rank test. LLOQ = lower limit of quantification (only depicted in graphs with measurements below LLOQ).

Table 1. Changes in biomarker levels in dialysates and biopsies $6 \mathrm{~h}$ after skin barrier disruption.

\begin{tabular}{ccc}
\hline Biomarker & Dialysates $^{\mathbf{1}}$ & Biopsies $^{\mathbf{1}}$ \\
\hline CCL27/CTACK & $\leftrightarrow$ & $\leftrightarrow$ \\
GM-CSF & $\uparrow$ & BLOQ \\
CXCL1/GRO $\alpha$ & $\uparrow$ & $\uparrow$ \\
CXCL8/IL-8 & $\uparrow$ & $\uparrow$ \\
CXCL10/IP-10 & $\uparrow$ & BLOQ \\
IL-1 $\alpha$ & $\uparrow$ & $(\downarrow)$ \\
IL-6 & $\uparrow$ & $\uparrow$ \\
MIF & $\uparrow$ & $\leftrightarrow$ \\
TNF- $\alpha$ & $\uparrow$ & BLOQ \\
VEGF & $\uparrow$ & BLOQ \\
\hline
\end{tabular}

$1 \uparrow$ : Significantly upregulated, $\downarrow$ : significantly downregulated, $(\downarrow)$ : a trend toward downregulation, $\leftrightarrow$ : no significant change, BLOQ: below lower limit of quantification.

The impact of skin-barrier disruption on the biomarker levels detected in dialysates and skin biopsy homogenates is summarized in Table 1. 
a

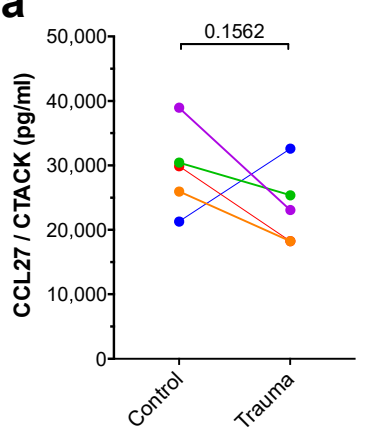

d

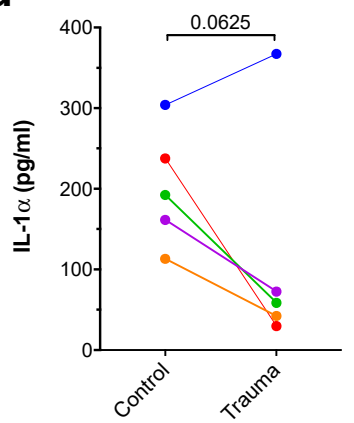

b

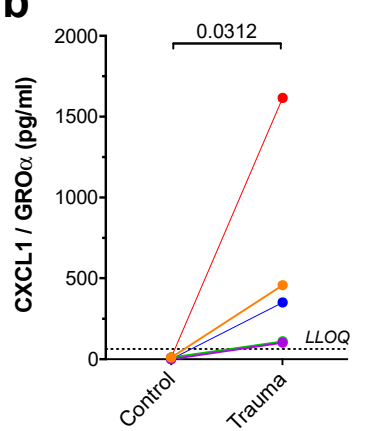

e

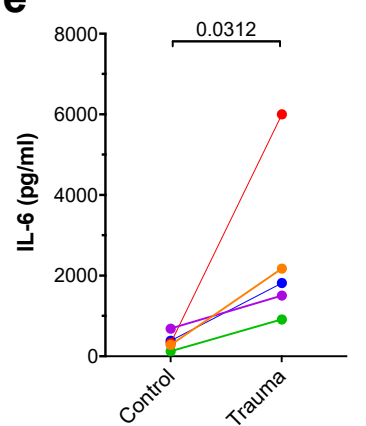

C

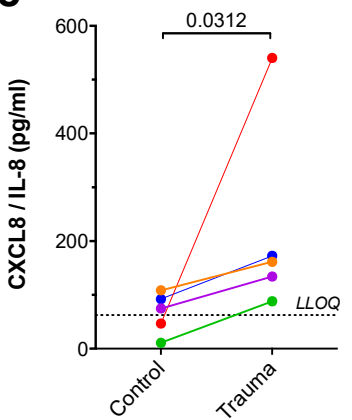

f

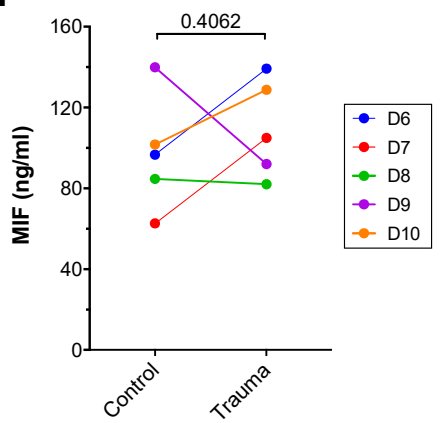

Figure 4. Total tissue levels of (a) CCL27/CTACK, (b) CXCL1/GRO $\alpha$, (c) CXCL8/IL-8, (d) IL-1 $\alpha$, (e) IL-6, and (f) MIF measured $6 \mathrm{~h}$ post abrasion in skin biopsy homogenates by ELISA, normalized to total protein content. Data are depicted for biopsies obtained from barrier-disrupted skin specimens (trauma) or control specimens from five individual skin donors after $6 \mathrm{~h}$ of incubation. Numbers above the bars indicate $p$-values obtained from Wilcoxon matched-pairs signed rank test. LLOQ = lower limit of quantification (only depicted in graphs with measurements below LLOQ).

\section{Discussion}

Prior to initiation of the experimental work presented in this paper, validation experiments assessing sampling efficiency of the 10 target analytes were performed [21]. The relative skin recoveries measured were all above the feasibility threshold of $4-5 \%$ [22] with the exception of CXCL10/IP-10 [21]. Despite the low relative skin recovery of CXCL10/IP-10 (0.9\%), the biomarker was included in the extended biomarker panel since the increased sensitivity of the MSD platform compared with ELISA was expected to enable successful detection [23,24].

Corresponding to previous reports, an overall increase in extracellular levels of the 10 biomarkers was observed both in the proof-of-concept study and in the main experiments after abrasive disruption of the skin barrier ex vivo. In line with our findings, a rapid release of GM-CSF following tape stripping or chemical perturbation of the skin barrier has been described by other groups $[17,25,26]$, as has the release of CXCL1/GRO $\alpha$, CXCL8/IL-8, CXCL10/IP-10, and TNF- $\alpha$ in both mice and humans $[3,17,25,27-30]$. Similarly, upregulation of another trauma-associated marker, IL-6, has been reported in response to human and murine skin-barrier perturbation [17,31,32]. VEGF is known to play a role in the later angiogenic phase of wound healing, but there are several studies showing an immediate secretion of the growth factor as a consequence of skin-barrier disruption [33-35], which is corroborated by the present study. The proinflammatory cytokine IL- $1 \alpha$ is prestored in keratinocytes and serves as an acute damage signal $[36,37]$. This is reflected by the increasing dialysate concentrations of IL- $1 \alpha$ observed in this study after mechanical disruption of the skin barrier. The decrease in IL-1 $\alpha$ to near-basal levels $24 \mathrm{~h}$ post abrasion has also been reported by other groups performing physical and chemical barrier disruption $[28,36]$. In agreement with the literature, we observed relatively high extracellular levels of the ubiquitously expressed [38] skin cytokine MIF in control 
skin specimens [39,40], and the cytokine was clearly upregulated in response to barrier perturbation. Conversely, levels of CCL27/CTACK were not affected by the abrasive treatment, despite previous reports of an upregulation in wounded skin [41,42]. A constitutive dermal expression of CCL27/CTACK [41,42] may explain our results if probe insertion mediates a release from damaged cells, which could potentially mask a slight increase in CCL27/CTACK secretion following skin-barrier disruption. It is also possible that the discrepancy between previous reports and levels of CCL27/CTACK observed in this study is a matter of kinetics, since only a single timepoint was assessed. Hence, microdialysis may be used in future studies to investigate the dynamic nature of biomarker release by performing continuous sampling to obtain a kinetic profile.

When comparing dialysate concentrations with the biomarker levels measured in homogenized skin biopsies, the effect of skin-barrier disruption was similar for CCL27/CTACK, CXCL1/GRO $\alpha$, CXCL8/IL-8, and IL-6 using the two approaches. However, the clear increase in dialysate levels of MIF following skin-barrier disruption was not mirrored by the total tissue levels, as no significant change in MIF concentrations was seen in the biopsy homogenates. This could be explained by the fact that skin biopsy extracts reflect both intra- and extracellular biomarker concentrations, whereas microdialysis sampling reveals changes in the extracellular biomarker levels. Since MIF is pre-formed in the skin [38], the intracellular levels may mask the amount released into the extracellular space upon disruption of the skin barrier. Likewise, the IL- $1 \alpha$ response to skin-barrier disruption appeared opposite using the two methods; dialysate concentrations were increased in barrier disrupted skin, whereas the total tissue levels determined from homogenized skin biopsies seemed to decrease in response to abrasive skin trauma. The drop in extracellular IL- $1 \alpha$ levels over time, which was observed in the proof-of-concept experiments, could indicate a breakdown of the cytokine once it is released from the cells. This might account for the decrease in total tissue levels; if the amount of IL- $1 \alpha$ released in response to skin barrier is metabolized faster than the intracellular stores are replenished, the total tissue content will decrease. This potential explanation points to an advantage of microdialysis, despite its methodological challenges (reviewed in [6]); this technique enables quantification of the extracellular biomarker levels, which are in fact determining the skin response, as opposed to biopsy homogenates portraying a combination of intra- and extracellular levels. However, the use of skin biopsies may be a suitable approach if the biomarker of interest cannot be recovered in sufficient amounts by microdialysis; either due to sampling-related issues caused by the physicochemical characteristics of the target analyte or due to analytical issues related to sample volume or detection assay sensitivity [6].

For the expanded biomarker profiling in this proof-of-concept study, the $6 \mathrm{~h}$ timepoint was chosen to allow for de novo synthesis of the relevant biomarkers, but earlier timepoints could also be investigated in future studies to assess the release of preformed mediators. Comparing the 6 and $24 \mathrm{~h}$ timepoints, we found a spontaneous increase in background levels of IL-6, which could indicate a decrease in skin viability over time, despite histological assessments indicating that tissue integrity and viability was well maintained within the first $24 \mathrm{~h}$ of incubation (Figures S1 and S2). Döge et al. also reported increasing background levels over time [28], and, while there was still a significant effect of the abrasive treatment at the later time point, we decided to focus on the $6 \mathrm{~h}$ timepoint for the main experiments due to a better signal-to-noise ratio. Furthermore, the use of excised skin involves the absence of blood flow, which leads to a deficient supply of oxygen and nutrients to the tissue, as well as a lack of cellular recruitment. The latter is well known to take place as part of the acute response to skin trauma; however, a previous study did not report of cellular infiltration in mechanically disrupted human skin within the first $6 \mathrm{~h}$ post barrier disruption [27]. Thus, we believe that the ex vivo model is still valid and providing valuable information within this time frame.

An increase in trauma-associated biomarkers following probe insertion has been described by other groups [43,44]. The low background dialysate levels measured in this study indicate that the insertion trauma is insignificant compared with the increase caused 
by abrasive disruption of the skin barrier. Yet, appropriate negative controls must always be included as this will reveal any background signal that may otherwise be interpreted as a direct effect of the abrasive procedure.

We observed a notable intersubject variability in biomarker levels, which has also been described by other groups [45-47]. This variation is likely caused by a combination of several factors such as differences in skin thickness, the abrasive procedure, intradermal membrane insertion, and general biological differences across individuals. Our data revealed that the intersubject variability exceeded the intrasubject variability (being the variation between replicate probes), in keeping with previous reports $[47,48]$. In future studies, assessment of intradermal membrane positioning using ultrasound and histological evaluation of the impact of the abrasive procedure could be used to control for technical variations across individual skin specimens. A limitation of the present study is the low number of skin donors, but the experimental approach may still be applied to future studies, e.g., as a preclinical tool to evaluate the efficacy of products designed to accelerate barrier repair. Our findings highlight the importance of including a sufficient number of skin donors in future studies.

\section{Conclusions}

In conclusion, the results presented in this paper indicate that microdialysis sampling of biomarkers from the interstitial fluid of human ex vivo skin specimens could be a useful tool to study the acute events following skin-barrier disruption. Subsequent sample analysis using a multiplex platform adds significant value to this approach. We suggest that this model may be used in future studies to investigate additional biomarkers implicated in skin-barrier restoration and to assess the effect of novel treatments intended for skinbarrier disorders.

Supplementary Materials: The following are available online at https://www.mdpi.com/article/10.3 390/dermato1020008/s1, Figure S1: Changes in skin morphology in response to incubation time, Figure S2: Changes in Ki67 expression in response to incubation time.

Author Contributions: Conceptualization, K.B., P.S.S. and A.W.; methodology, K.B., N.P.H.K. and P.S.S.; software, K.B. and N.P.H.K.; validation, K.B. and N.P.H.K.; formal analysis, K.B. and N.P.H.K.; investigation, K.B., N.P.H.K. and A.-S.Ø.G.; resources, P.S.S., A.-S.Ø.G. and A.W.; data curation, K.B. and N.P.H.K.; writing — original draft preparation, K.B. and P.S.S.; writing—review and editing K.B., N.P.H.K., P.S.S., A.-S.Ø.G. and A.W.; visualization, K.B. and N.P.H.K.; supervision, K.B. and P.S.S.; project administration, K.B., N.P.H.K. and P.S.S.; funding acquisition, K.B., P.S.S. and A.W. All authors have read and agreed to the published version of the manuscript.

Funding: K.B. was supported by Innovation Fund Denmark ("Innovationsfonden") through an industrial Ph.D. scholarship (5189-00012B) covering part of her salary. Otherwise, this research received no external funding.

Institutional Review Board Statement: Ethical review and approval were waived for this study, due to the use of fully anonymized tissue according to the Danish Act on Research Ethics Review of Health Research Projects, Section 14 (3) concerning the use of anonymized human material for research purposes.

Informed Consent Statement: Patient consent was waived due to the use of fully anonymized tissue according to the Danish Act on Research Ethics Review of Health Research Projects, Section 14 (3) concerning the use of anonymized human material for research purposes.

Data Availability Statement: All data needed to evaluate the conclusions made in this paper are included in the main body of the manuscript. Additional data will be made available by the corresponding author upon reasonable request.

Acknowledgments: The authors thank Nicole van der Burg for excellent technical assistance. UPM Biomedicals is acknowledged for supporting the study. 
Conflicts of Interest: K.B. was employed by RefLab ApS as Industrial PhD student through a fellowship funded by Innovation Fund Denmark when this work was conducted. The funders had no role in the design of the study; in the collection, analyses, or interpretation of data; in the writing of the manuscript, or in the decision to publish the results. N.P.H.K. is a former employee at RefLab ApS. P.S.S. is acting as research consultant for RefLab ApS and EP Medical. The other authors declare no conflict of interest.

\section{References}

1. Proksch, E.; Brandner, J.M.; Jensen, J.-M. The skin: An indispensable barrier. Exp. Dermatol. 2008, 17, 1063-1072. [CrossRef] [PubMed]

2. Hänel, K.; Cornelissen, C.; Lüscher, B.; Baron, J. Cytokines and the Skin Barrier. Int. J. Mol. Sci. 2013, 14, 6720-6745. [CrossRef]

3. Engelhardt, E.; Toksoy, A.; Goebeler, M.; Debus, S.; Bröcker, E.-B.; Gillitzer, R. Chemokines IL-8, GRO $\alpha$, MCP-1, IP-10, and Mig Are Sequentially and Differentially Expressed during Phase-Specific Infiltration of Leukocyte Subsets in Human Wound Healing. Am. J. Pathol. 1998, 153, 1849-1860. [CrossRef]

4. Antonov, D.; Schliemann, S.; Elsner, P. Methods for the Assessment of Barrier Function. Skin Barrier Funct. 2016, 49, 61-70. [CrossRef]

5. Gao, Y.; Wang, X.; Chen, S.; Li, S.; Liu, X. Acute skin barrier disruption with repeated tape stripping: Anin vivomodel for damage skin barrier. Ski. Res. Technol. 2013, 19, 162-168. [CrossRef] [PubMed]

6. $\quad$ Baumann, K.Y.; Church, M.K.; Clough, G.F.; Quist, S.R.; Schmelz, M.; Skov, P.S.; Anderson, C.D.; Tannert, L.K.; Giménez-Arnau, A.M.; Frischbutter, S.; et al. Skin microdialysis: Methods, applications and future opportunities-An EAACI position paper. Clin. Transl. Allergy 2019, 9, 24. [CrossRef] [PubMed]

7. Rea, H.; Kirby, B. A Review of Cutaneous Microdialysis of Inflammatory Dermatoses. Acta Derm. Venereol. 2019, 99, 945-952 [CrossRef]

8. Sjögren, F.; Anderson, C.D. Are Cutaneous Microdialysis Cytokine Findings Supported by End Point Biopsy Immunohistochemistry Findings? AAPS J. 2010, 12, 741-749. [CrossRef]

9. Plock, N.; Kloft, C. Microdialysis-Theoretical background and recent implementation in applied life-sciences. Eur. J. Pharm. Sci. 2005, 25, 1-24. [CrossRef]

10. Ud-Din, S.; Bayat, A. Non-animal models of wound healing in cutaneous repair: In silico, in vitro, ex vivo, and in vivo models of wounds and scars in human skin. Wound Repair Regen. 2017, 25, 164-176. [CrossRef]

11. Bartek, M.J.; Labudde, J.A.; Maibach, H.I. Skin Permeability In Vivo: Comparison in Rat, Rabbit, Pig and Man. J. Investig. Dermatol. 1972, 58, 114-123. [CrossRef] [PubMed]

12. Godin, B.; Touitou, E. Transdermal skin delivery: Predictions for humans from in vivo, ex vivo and animal models. Adv. Drug Deliv. Rev. 2007, 59, 1152-1161. [CrossRef]

13. Danso, M.O.; Berkers, T.; Mieremet, A.; Hausil, F.; Bouwstra, J.A. Anex vivo humanskin model for studying skin barrier repair. Exp. Dermatol. 2015, 24, 48-54. [CrossRef]

14. Berkers, T.; Boiten, W.; Absalah, S.; van Smeden, J.; Lavrijsen, A.; Bouwstra, J. Compromising human skin in vivo and ex vivo to study skin barrier repair. Biochim. Biophys. Acta (BBA) Mol. Cell Biol. Lipids 2019, 1864, 1103-1108. [CrossRef]

15. Berkers, T.; Visscher, D.; Gooris, G.; Bouwstra, J. Degree of Skin Barrier Disruption Affects Lipid Organization in Regenerated Stratum Corneum. Acta Derm. Venereol. 2018, 98, 421-427. [CrossRef]

16. Döge, N.; Hönzke, S.; Schumacher, F.; Balzus, B.; Colombo, M.; Hadam, S.; Rancan, F.; Blume-Peytavi, U.; Schäfer-Korting, M.; Schindler, A.; et al. Ethyl cellulose nanocarriers and nanocrystals differentially deliver dexamethasone into intact, tape-stripped or sodium lauryl sulfate-exposed ex vivo human skin-Assessment by intradermal microdialysis and extraction from the different skin layers. J. Control. Release 2016, 242, 25-34. [CrossRef]

17. Pfannes, E.K.; Weiss, L.; Hadam, S.; Gonnet, J.; Combardiere, B.; Blume-Peytavi, U.; Vogt, A. Physiological and Molecular Effects of in vivo and ex vivo Mild Skin Barrier Disruption. Ski. Pharmacol. Physiol. 2018, 31, 115-124. [CrossRef]

18. Kidwell, M.J.; Arpey, C.J.; Messingham, M.J. A Comparison of Histologic Effectiveness and Ultrastructural Properties of the Electrocautery Scratch Pad to Sandpaper for Manual Dermabrasion. Dermatol. Surg. 2008, 34, 1194-1199. [CrossRef]

19. Pavlidis, L.; Spyropoulou, G.-A. A Simple Technique to Perform Manual Dermabrasion with Sandpaper. Dermatol. Surg. 2012, 38, 2016-2017. [CrossRef] [PubMed]

20. Wu, X.-M.; Todo, H.; Sugibayashi, K. Effects of pretreatment of needle puncture and sandpaper abrasion on the in vitro skin permeation of fluorescein isothiocyanate (FITC)-dextran. Int. J. Pharm. 2006, 316, 102-108. [CrossRef] [PubMed]

21. Baumann, K.; Falkencrone, S.; Knudsen, N.P.; Woetmann, A.; Dabelsteen, S.; Skov, P.S. The Skin Reservoir Model: A Tool for Evaluating Microdialysis Sampling of Large Biomarkers from Human Skin. Acta Derm. Venereol. 2019, 100, 1-8. [CrossRef]

22. Holmgaard, R.; Nielsen, J.; Benfeldt, E. Microdialysis Sampling for Investigations of Bioavailability and Bioequivalence of Topically Administered Drugs: Current State and Future Perspectives. Ski. Pharmacol. Physiol. 2010, 23, 225-243. [CrossRef] [PubMed]

23. Stenken, J.A.; Poschenrieder, A. Bioanalytical chemistry of cytokines-A review. Anal. Chim. Acta 2015, 853, 95-115. [CrossRef] [PubMed] 
24. Dabitao, D.; Margolick, J.B.; Lopez, J.; Bream, J.H. Multiplex measurement of proinflammatory cytokines in human serum: Comparison of the Meso Scale Discovery electrochemiluminescence assay and the Cytometric Bead Array. J. Immunol. Methods 2011, 372, 71-77. [CrossRef]

25. Wood, L.C.; Jackson, S.M.; Elias, P.M.; Grunfeld, C.; Feingold, K.R. Cutaneous barrier perturbation stimulates cytokine production in the epidermis of mice. J. Clin. Investig. 1992, 90, 482-487. [CrossRef] [PubMed]

26. Schirmacher, P.; Mann, A.; Breuhahn, K.; Blessing, M. Keratinocyte-Derived Granulocyte-Macrophage Colony Stimulating Factor Accelerates Wound Healing: Stimulation of Keratinocyte Proliferation, Granulation Tissue Formation, and Vascularization. J. Investig. Dermatol. 2001, 117, 1382-1390. [CrossRef] [PubMed]

27. Nickoloff, B.J.; Naidu, Y. Perturbation of epidermal barrier function correlates with initiation of cytokine cascade in human skin. J. Am. Acad. Dermatol. 1994, 30, 535-546. [CrossRef]

28. Döge, N.; Avetisyan, A.; Hadam, S.; Pfannes, E.K.B.; Rancan, F.; Blume-Peytavi, U.; Vogt, A. Assessment of skin barrier function and biochemical changes of ex vivo human skin in response to physical and chemical barrier disruption. Eur. J. Pharm. Biopharm. 2017, 116, 138-148. [CrossRef]

29. Dickel, H.; Gambichler, T.; Kamphowe, J.; Altmeyer, P.; Skrygan, M. Standardized tape stripping prior to patch testing induces upregulation of Hsp90, Hsp70, IL-33, TNF- $\alpha$ and IL-8/CXCL8 mRNA: New insights into the involvement of 'alarmins'. Contact Dermat. 2010, 63, 215-222. [CrossRef]

30. Kroeze, K.L.; Boink, M.A.; Sampat-Sardjoepersad, S.C.; Waaijman, T.; Scheper, R.J.; Gibbs, S. Autocrine Regulation of ReEpithelialization After Wounding by Chemokine Receptors CCR1, CCR10, CXCR1, CXCR2, and CXCR3. J. Investig. Dermatol. 2012, 132, 216-225. [CrossRef]

31. Wang, X.-P.; Schunck, M.; Kallen, K.-J.; Neumann, C.; Trautwein, C.; Rose-John, S.; Proksch, E. The Interleukin-6 Cytokine System Regulates Epidermal Permeability Barrier Homeostasis. J. Investig. Dermatol. 2004, 123, 124-131. [CrossRef]

32. Grellner, W. Time-dependent immunohistochemical detection of proinflammatory cytokines (IL-1 $\beta$, IL-6, TNF- $\alpha$ ) in human skin wounds. Forensic Sci. Int. 2002, 130, 90-96. [CrossRef]

33. Brown, L.F.; Yeo, K.T.; Berse, B.; Yeo, T.K.; Senger, D.R.; Dvorak, H.F.; Van De Water, L. Expression of vascular permeability factor (vascular endothelial growth factor) by epidermal keratinocytes during wound healing. J. Exp. Med. 1992, 176, 1375-1379. [CrossRef]

34. Nissen, N.N.; Polverini, P.J.; Koch, A.E.; Volin, M.V.; Gamelli, R.L.; DiPietro, L.A. Vascular endothelial growth factor mediates angiogenic activity during the proliferative phase of wound healing. Am. J. Pathol. 1998, 152, 1445-1452.

35. Elias, P.M.; Arbiser, J.; Brown, B.E.; Rossiter, H.; Man, M.-Q.; Cerimele, F.; Crumrine, D.; Gunathilake, R.; Choi, E.H.; Uchida, Y.; et al. Epidermal Vascular Endothelial Growth Factor Production Is Required for Permeability Barrier Homeostasis, Dermal Angiogenesis, and the Development of Epidermal Hyperplasia: Implications for the Pathogenesis of Psoriasis. Am. J. Pathol. 2008, 173, 689-699. [CrossRef]

36. Wood, L.C.; Elias, P.M.; Calhoun, C.; Tsai, J.C.; Grunfeld, C.; Feingold, K.R. Barrier Disruption Stimulates Interleukin-1 $\alpha$ Expression and Release from a Pre-Formed Pool in Murine Epidermis. J. Investig. Dermatol. 1996, 106, 397-403. [CrossRef] [PubMed]

37. Lee, R.T.; Briggs, W.H.; Cheng, G.C.; Rossiter, H.B.; Libby, P.; Kupper, T. Mechanical deformation promotes secretion of IL-1 alpha and IL-1 receptor antagonist. J. Immunol. 1997, 159, 5084-5088.

38. Gilliver, S.C.; Emmerson, E.; Bernhagen, J.; Hardman, M.J. MIF: A key player in cutaneous biology and wound healing. Exp. Dermatol. 2010, 20,1-6. [CrossRef]

39. Grieb, G.; Simons, D.; Eckert, L.; Hemmrich, M.; Steffens, G.; Bernhagen, J.; Pallua, N. Levels of macrophage migration inhibitory factor and glucocorticoids in chronic wound patients and their potential interactions with impaired wound endothelial progenitor cell migration. Wound Repair Regen. 2012, 20, 707-714. [CrossRef] [PubMed]

40. Abe, R.; Shimizu, T.; Ohkawara, A.; Nishihira, J. Enhancement of macrophage migration inhibitory factor (MIF) expression in injured epidermis and cultured fibroblasts. Biochim. Biophys. Acta (BBA) Mol. Basis Dis. 1999, 1500, 1-9. [CrossRef]

41. Bünemann, E.; Hoff, N.-P.; Buhren, B.A.; Wiesner, U.; Meller, S.; Bölke, E.; Müller-Homey, A.; Kubitza, R.; Ruzicka, T.; Zlotnik, A.; et al. Chemokine ligand-receptor interactions critically regulate cutaneous wound healing. Eur. J. Med Res. 2018, 23, 4. [CrossRef]

42. Inokuma, D.; Abe, R.; Fujita, Y.; Sasaki, M.; Shibaki, A.; Nakamura, H.; McMillan, J.R.; Shimizu, T.; Shimizu, H. CTACK/CCL27 Accelerates Skin Regeneration via Accumulation of Bone Marrow-Derived Keratinocytes. Stem Cells 2006, 24, $2810-2816$. [CrossRef] [PubMed]

43. Sjögren, F.; Anderson, C. Sterile Trauma to Normal Human Dermis Invariably Induces IL1beta, IL6 and IL8 in an Innate Response to "Danger". Acta Derm. Venereol. 2009, 89, 459-465. [CrossRef]

44. Stenken, J.A.; Church, M.K.; Gill, C.A.; Clough, G.F. How Minimally Invasive is Microdialysis Sampling? A Cautionary Note for Cytokine Collection in Human Skin and other Clinical Studies. AAPS J. 2010, 12, 73-78. [CrossRef] [PubMed]

45. Perkins, M.A.; Osterhues, M.A.; Farage, M.A.; Robinson, M.K. A noninvasive method to assess skin irritation and compromised skin conditions using simple tape adsorption of molecular markers of inflammation. Ski. Res. Technol. 2001, 7, 227-237. [CrossRef] [PubMed] 
46. Benfeldt, E.; Hansen, S.H.; Vølund, A.; Menné, T.; Shah, V.P. Bioequivalence of Topical Formulations in Humans: Evaluation by Dermal Microdialysis Sampling and the Dermatopharmacokinetic Method. J. Investig. Dermatol. 2007, 127, 170-178. [CrossRef] [PubMed]

47. Tettey-Amlalo, R.N.O.; Kanfer, I.; Skinner, M.F.; Benfeldt, E.; Verbeeck, R.K. Application of dermal microdialysis for the evaluation of bioequivalence of a ketoprofen topical gel. Eur. J. Pharm. Sci. 2009, 36, 219-225. [CrossRef] [PubMed]

48. Benfeldt, E.; Serup, J.; Menné, T. Microdialysis vs. Suction Blister Technique for In vivo Sampling of Pharmacokinetics in the Human Dermis. Acta Derm. Venereol. 1999, 79, 338-342. [CrossRef] 